\title{
Poly(lactic-co-glycolic acid)-bioactive glass composites as nanoporous scaffolds for bone tissue engineering: In vitro and in vivo studies
}

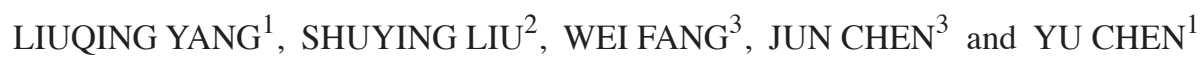 \\ ${ }^{1}$ Department of Stomatology, Guangzhou Women and Children's Medical Center, Guangzhou, Guangdong 510623; \\ Departments of ${ }^{2}$ Periodontics and ${ }^{3}$ Oral and Maxillofacial Surgery, Stomatological Hospital, \\ Southern Medical University, Guangzhou, Guangdong 510280, P.R. China
}

Received February 1, 2019; Accepted September 27, 2019

DOI: $10.3892 /$ etm.2019.8121

\begin{abstract}
The aim of the present study was to investigate the feasibility of using composite scaffolds of poly(lactic-co-glycolic acid) (PLGA) and bioactive glass (BG) to repair bone defects. PLGA/BG composite scaffolds were prepared by thermally-induced phase separation. Scanning electron microscopy (SEM) was used to study the morphology, and liquid (absolute ethanol) replacement was used to calculate the porosity of the scaffold. The biocompatibility and degradation of the scaffold were determined using human osteosarcoma cell line MG-63 and animal experiments. SEM showed that the scaffold had a nanofibrous three-dimensional network structure with a fiber diameter of 160-320 nm, a pore size of $1-7 \mu \mathrm{m}$, and a porosity of $93.048 \pm 0.121 \%$. The scaffold structure was conducive to cell adhesion and proliferation. It promoted cell osteogenesis and could be stably degraded in vivo.
\end{abstract}

\section{Introduction}

To date, clinical studies have shown that large defects of the jaw due to traumatic injury or tumor resection do not heal themselves (1). Clinically, autologous bone, allogeneic bone and xenografts are often used to repair bone defects. However, these treatments have their drawbacks, including limited availability, infection in the donor area and immune rejection (2). Modern tissue engineering proposes a new direction for the treatment of bone defects, by designing a porous biomimetic natural extracellular matrix (ECM) scaffold, to provide cells with a microenvironment for growth, proliferation and differentiation. Compared to the ECM scaffold, the

Correspondence to: Dr Shuying Liu, Department of Periodontics, Stomatological Hospital, Southern Medical University, 366 Jiangnan Da Dao, Guangzhou, Guangdong 510280, P.R. China

E-mail: 1048784509@qq.com

Key words: poly(lactic-co-glycolic acid), scaffold, bioactive glass, bone tissue engineering high-porosity nanofiber scaffold provides more space for cells, facilitating the exchange of nutrients and oxygen and the excretion of metabolic waste (3). Degradable polymer scaffolds also play an important role in modern tissue engineering. If the implanted materials degrade too fast, before the new bone has formed, the scaffold will not be able to provide cells with a microenvironment for growth. However, if the materials are non-degradable after the implantation, and continue to remain in the form of foreign matter, the risk of infection is increased. It is therefore required that the degradation rate of bone scaffolds should match the formation rate of new bone and that the degradation products of the scaffolds are not toxic.

In recent years, polymer composite scaffolds as drug or gene delivery carriers have been widely used in tissue engineering (4). Poly(lactic-co-glycolic acid) (PLGA) is a degradable, functional compound with good biocompatibility and mechanical properties. It is widely used in pharmaceutical and medical engineering materials, and is certified by FDA $(5,6)$. Because PLGA lacks the ability to promote osteoblast differentiation, strategies are needed to combine it with other materials. Bioactive glass (BG) is a material that can repair, replace and regenerate body tissues, and can form a bond between tissues and materials (7). The degradation products of BG can promote growth factor production, cell proliferation, gene expression of osteoblasts and growth of bone tissue $(8,9)$. Animal experiments have shown that a combination of BG and PLGA can accelerate bioceramic degradation and promote bone defect regeneration (10-13).

Although PLGA combined with BG has been reported to have a positive catalytic significance in bone healing in several studies (7,10-13), the long-term degradation effects of BG and PLGA-composite scaffolds in vivo have not been extensively studied. In the present study, a PLGA/BG composite scaffold was prepared by thermal phase separation. The purpose of the present study was to evaluate the characteristics and physicochemical properties of the composite scaffold. MG-63 cells were used to evaluate the in vitro biocompatibility of the scaffold. Scaffold degradation was assessed after subcutaneous transplant into New Zealand white rabbits. 


\section{Materials and methods}

Preparation of PLGA/BG composite scaffold. Micro-nano powders of BG (medical grade; donated by South China University of Technology) were added to $10 \mathrm{ml}$ tetrahydrofuran (analytical pure; Guangzhou Jinhua Chemical Reagent Co., Ltd.) and dispersed using ultrasonic oscillation (SCIENTZ-1500F; Ningbo Scientz Biotechnology Co., Ltd.). PLGA (medical grade; Shenzhen Guanghua Weiye Industrial Co., Ltd; PLGA:BG ratio, 9:1) was then weighed and added to the tetrahydrofuran solution containing BG. The PLGA/BG solution was incubated in a $60^{\circ} \mathrm{C}$ water bath and stirred for $2 \mathrm{~h}$ until all powders had dissolved. The resulting PLGA/BG solution was transferred into a mold and immediately placed in a $-20^{\circ} \mathrm{C}$ freezer for at least $2 \mathrm{~h}$ to solidify into a gel. The gel was then transferred to $4^{\circ} \mathrm{C}$ for 1 day of water displacement, followed by incubation in a Genesis freeze dryer (VirTis; SP Scientific; $-55^{\circ} \mathrm{C}$, pressure $<50 \mathrm{~Pa}$ ) to lyophilize for $48 \mathrm{~h}$ to obtain the PLGA/BG nanofiber composite scaffold.

Analysis of surface morphology. The surface morphology of the composite scaffold was evaluated by scanning electron microscopy (SEM). A 4x4 mm composite scaffold was adhered to a copper substrate, and sprayed with gold under a vacuum. A JSM-6330F cold cathode field emission SEM (JEOL, Ltd.; provided by Sun Yat-sen University Test Center) was used to analyze the surface morphology of the gold-coated PLGA/BG composite scaffold.

Determination of porosity. A liquid (absolute ethanol) replacement method was used to measure porosity. The proportion pycnometer was filled with ethanol (analytically pure; Sinopharm Chemical Reagent Co., Ltd.) to a value of $\mathrm{W}_{1}(\mathrm{~g})$. The sample [with the quality set as $\mathrm{W}_{\mathrm{S}}(\mathrm{g})$ ] was immersed in the pycnometer filled with ethanol to de-aerate and allow the ethanol to fill the membrane's pores, followed by replacement of the ethanol. The value of the pycnometer containing the sample and the ethanol was defined as $\mathrm{W}_{2}(\mathrm{~g})$. After removing the sample from the ethanol, the value of the pycnometer containing the remaining ethanol was defined as $\mathrm{W}_{3}(\mathrm{~g})$.

The porosity of the sample scaffold was calculated based on the following equation. Here, $P$ represents the porosity (\%) of the material; $\mathrm{V}_{0}$ is the volume of material under normal conditions, named as the apparent volume $\left(\mathrm{cm}^{3}\right)$; and $V$ represents the volume of compacted material $\left(\mathrm{cm}^{3}\right)$.

$$
P=\frac{V_{0}-V}{V_{0}} \times 100 \%=\frac{W_{2}-W_{3}-W_{5}}{W_{1}-W_{3}} \times 100 \%
$$

Biocompatibility and osteogenic properties of a PLGA/BG composite scaffold. MG-63 osteosarcoma cell culture. Human MG-63 osteosarcoma cells (Nanjing Kehao Biotechnology Co., Ltd.) were transferred to a culture flask containing $10 \%$ fetal bovine serum (Beijing Solarbio Science \& Technology Co., Ltd.), $100 \mathrm{U} / \mathrm{ml}$ penicillin, and $100 \mu \mathrm{g} / \mathrm{ml}$ streptomycin in low-glucose DMEM (Beijing Solarbio Science \& Technology Co., Ltd.), which was then incubated at $37^{\circ} \mathrm{C}$ in a $5 \% \mathrm{CO}_{2}$ incubator. Once the cells reached $80 \%$ confluence, the culture medium was removed and the cells harvested using a $0.02 \%$ EDTA solution containing $0.25 \%$ trypsin. The number of cells was counted and the cell density adjusted to a 1:3 ratio for further subculture.

Measurement of alkaline phosphatase (ALP) activity of MG-63 cells on the composite scaffolds. A 100- $\mu 1$ cell suspension containing $1 \times 10^{5}$ MG-63 cells was seeded into a 24-well plate containing two groups of scaffold (PLGA only and PLGA/BG). The cell suspension was incubated with each scaffold for 3,7 or 14 days. Culture medium was then removed and plates rinsed 3 times with PBS, before cells were lysed with $200 \mu 1$ $1 \%$ Triton X-100 solution for $10-15 \mathrm{~min}$ at $4^{\circ} \mathrm{C}$. A $30-\mu 1$ solution was removed from each well and added to a fresh 96-well plate for the ALP assay, using the ALP Assay kit (Beijing Solarbio Science \& Technology Co., Ltd.), according to the manufacturer's instructions. The absorbance of each well was measured at $520 \mathrm{~nm}$ and the optical density (OD) was calculated.

Observation of MG-63 cell morphology on a composite scaffold. The PLGA/BG composite scaffolds were pre-wetted in the cell culture solution as described above. Pre-wetted composite scaffolds were placed in a 24-well plate and $100 \mu \mathrm{l}$ of a cell suspension containing $1 \times 10^{4}$ MG-63 cells added to each well. The scaffolds were incubated in the cell suspension for 3 or 7 days, before removal of the culture medium and fixation of the cells with $2.5 \%$ pentanediol at $4^{\circ} \mathrm{C}$ overnight. The scaffolds were then dehydrated in graded ethanol, and coated with colloidal gold before cell adhesion, cell proliferation and calcium nodule formation were observed using SEM.

\section{Degradation experiments in vivo}

Animal model creation. All animal experiments in this study were approved by the Animal Ethical and Welfare Committee of Sun Yat-sen University (IACUC-DB-15-0502) and all experiments were conducted in accordance with their guidelines. Operations were performed with the animals under general anesthesia using an intravenous injection of $30 \mathrm{mg} / \mathrm{kg}$ 3\% sodium pentobarbital (Wuhan Dongkang Source Technology Co., Ltd). Nine female New Zealand white rabbits aged 7-8 weeks were obtained from Guangdong Medical Laboratory Animal Center and one transplant was performed on each rabbit ear. A transverse incision was made on the appropriate area of the rabbit ear, and a subcutaneous pocket was created between the rabbit ear epidermis and the cartilage. The PLGA/BG scaffolds were implanted in the pockets, and the incisions were closed with sutures. Rabbits were sacrificed after 2 weeks, or at 3 or 6 months post-surgery. Tissues from the surgical areas, limited to $\sim 3 \mathrm{~mm}$ outside the transplanted areas, of full ear thickness were obtained, with 6 tissue samples obtained per time period.

Histological analysis. Tissues were fixed in 10\% formalin at room temperature for $48 \mathrm{~h}$ and decalcified with EDTA decalcification solution at room temperature for 2 weeks. Tissues were then dehydrated in a gradient ethanol solution and embedded in paraffin using an automatic biological tissue-embedding machine TB-718 (Hubei Taiva Medical Technologies Co., Ltd.). Paraffin-embedded sections with a thickness of 5-7 $\mu \mathrm{m}$ were obtained after sectioning using a rotary microtome (Leica Microsystems Shanghai Ltd.). For hematoxylin and eosin (H\&E) staining at room temperature, 

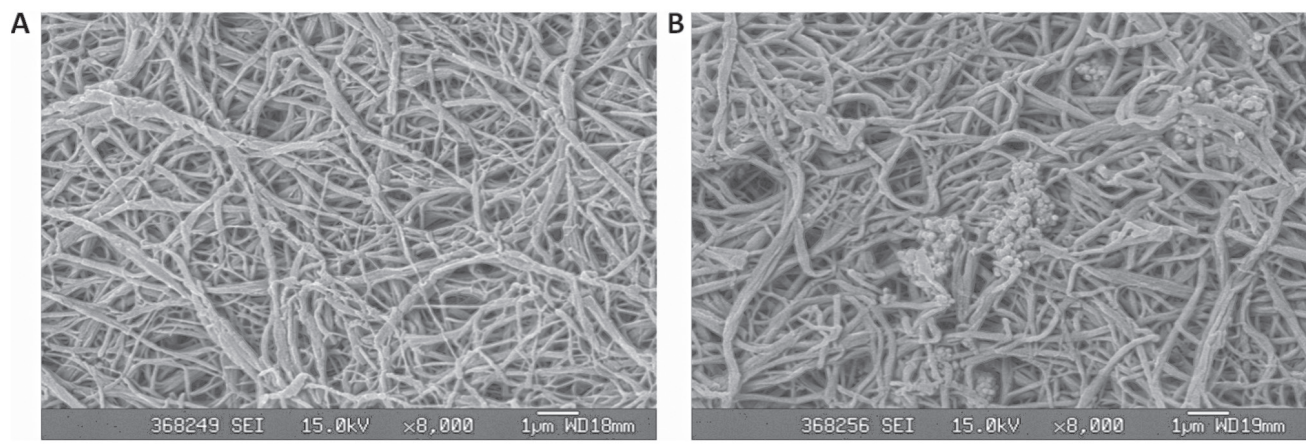

Figure 1. Representative SEM images of a scaffold (magnification, $x 8,000$ ). (A) SEM image of the PLGA scaffold. (B) SEM image of the PLGA/BG composite scaffold. BG, bioactive glass; PLGA, poly(lactic co-glycolic acid); SEM, scanning electron microscopy.

paraffin-embedded sections were routinely de-waxed, soaked for $15 \mathrm{~min}$ in hematoxylin, and rinsed of excess stain under running water. Sections were then soaked in $1 \%$ hydrochloric acid ethanol for $5 \mathrm{sec}$, rinsed under running water for $15 \mathrm{~min}$ and soaked in eosin dye solution for $5 \mathrm{~min}$. The sections then underwent gradient alcohol dehydration and were sealed with neutral gum, before they were observed under an ordinary light microscope (optical microscope BX41TF; Olympus Corporation). Four H\&E-stained paraffin slices from the same surgical region of each sample were randomly selected at 2 weeks, 3 months and 6 months. At x 400 magnification, 5 fields of view were randomly selected and images gathered of each slice of the transplanted area.

For Sirius red staining, paraffin-embedded sections were routinely de-waxed and stained with Sirius red droplets (Beijing Leagene Biotech. Co., Ltd.) at room temperature for $1 \mathrm{~h}$. Sections were then rinsed under running water and Mayer's hematoxylin staining solution was used to stain nuclei at room temperature for $10 \mathrm{~min}$. The stained sections underwent graded alcohol dehydration and were sealed with neutral gum before they were viewed under a polarized light microscope (STEMI 2000; Zeiss GmbH). At x200 magnification, three fields of view were randomly selected and images gathered of each slice of the transplanted area.

Statistical analysis. SPSS 19.0 software (IBM Corp.) was used for statistical analyses. All measurement data are presented as the mean $\pm \mathrm{SD}$. A one-way analysis of variance was used for data analysis. LSD was used as the post hoc test. $\mathrm{P}<0.05$ was considered to indicate a statistically significant difference.

\section{Results}

Micromorphology of the composite scaffolds. As shown in Fig. 1A, the PLGA scaffold generated from experimentally-induced phase separation formed a three-dimensional network structure. The fiber material diameter was $160-320 \mathrm{~nm}$ with a uniform pore distribution and pore sizes of 1-7 $\mu \mathrm{m}$. Fig. 1B shows that BG particles adhered to the PLGA matrix and dispersed in the porous material. The fiber material diameter was $160-320 \mathrm{~nm}$ with a uniform pore distribution and pore sizes of $1-7 \mu \mathrm{m}$. The fiber diameter and porosity of the composite material showed no obvious change in comparison to the scaffold generated with PLGA alone. The two scaffolds formed nano-diameter fibers with micron-sized pores.

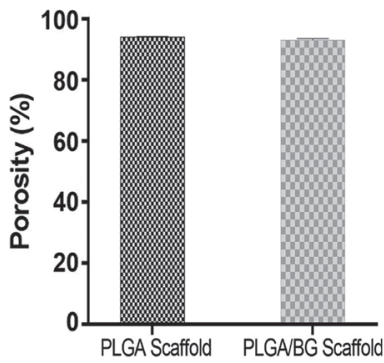

Figure 2. Comparison of porosity between PLGA only scaffolds and PLGA/BG scaffolds. BG, bioactive glass; PLGA, poly(lactic co-glycolic acid).

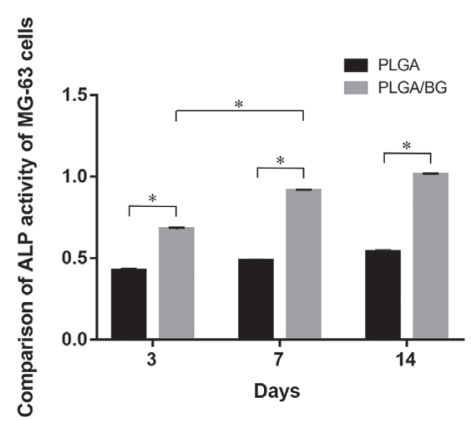

Figure 3. Comparison of ALP activity of MG-63 cells in two groups "P $<0.01$. ALP, alkaline phosphatase; BG, bioactive glass; PLGA, poly (lactic co-glycolic acid).

Porosity of the composite scaffolds. The PLGA scaffold had a high porosity (93.926 $\pm 0.094 \%)$. The porosity of the PLGA/BG composite scaffold was $93.048 \pm 0.121 \%$, which was slightly lower, but in general the structure of the scaffold did not appear altered. There was no significant difference in porosity between the two groups and both groups showed a high porosity (Fig. 2).

Measurement of ALP activity of MG-63 cells. The overall ALP activity of MG-63 cells in each group appeared to increase over time. The OD value of cells on the PLGA/BG scaffold was higher than that of cells grown on the PLGA scaffold at each of three time points studied $(\mathrm{P}<0.01)$. The OD value of the cells grown on the PLGA/BG for 7 days was significantly higher than the OD value of the cells grown on the scaffold for 3 days $(\mathrm{P}<0.01)$. There was no significant difference in $\mathrm{OD}$ value between cells cultured on the PLGA/BG for 7 days and the cells cultured on the PLGA/BG scaffold for 14 days (Fig. 3). 

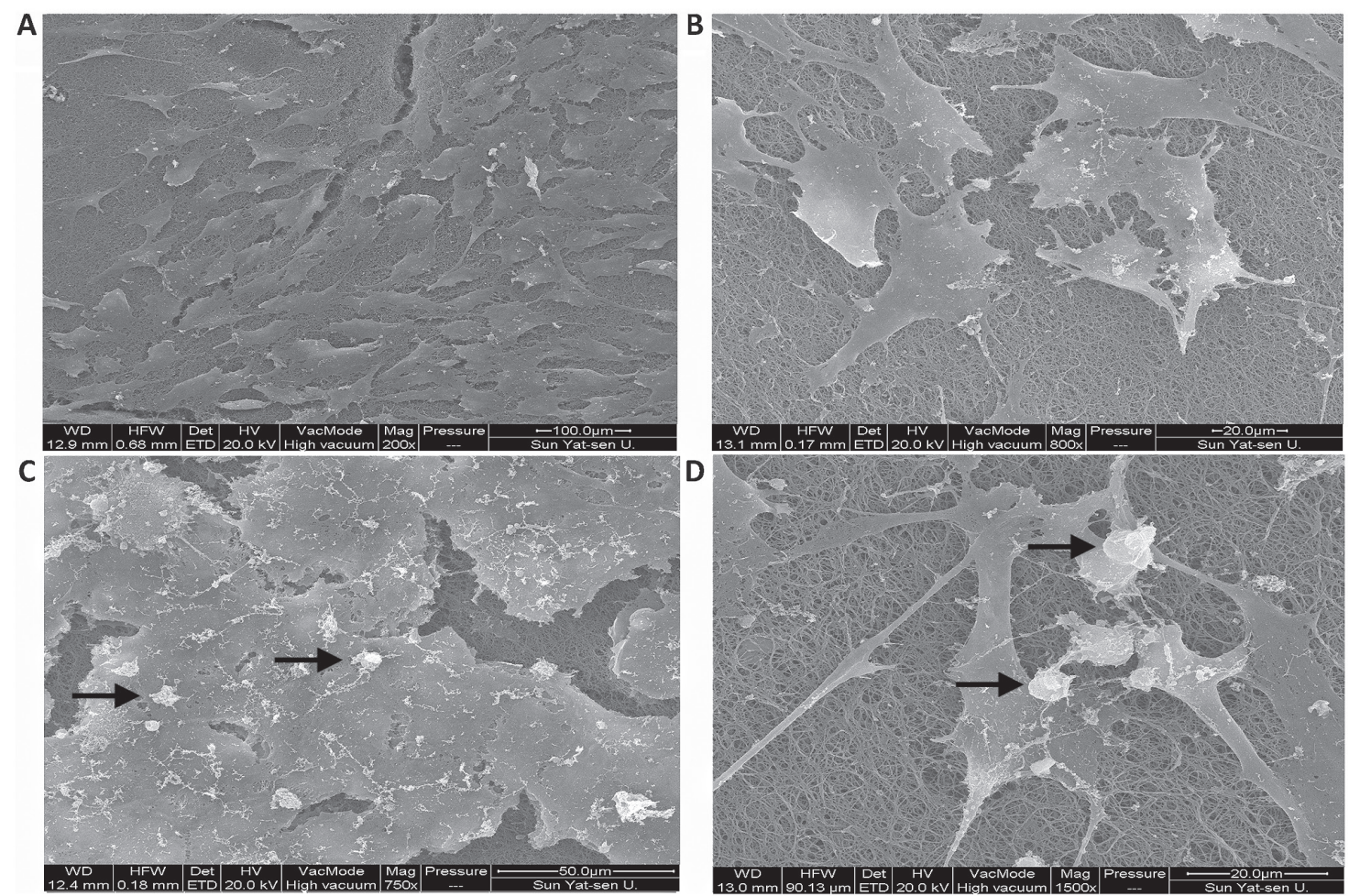

Figure 4. SEM images of MG-63 cells in the PLGA/BG scaffold. SEM image of MG-63 cells in PLGA/BG scaffold on the 3rd day at (A) x200 and (B) x800. SEM image of MG-63 cells in PLGA/BG scaffold on the 7th day at (C) x750 and (D) x1500. Arrows indicate calcified nodules. BG, bioactive glass; PLGA, poly(lactic co-glycolic acid); SEM, scanning electron microscopy.

Morphological observation of MG-63 cells. SEM data indicated that after 3 days of culture, MG-63 cells were spindle-shaped and adhered to the surface of the PLGA/BG composite scaffold. These cells began to extend pseudopodia in all directions to cross-link with the composite scaffold (Fig. 4A and B). After 7 days of culture, the MG-63 cells formed colonies after aggregating on multiple layers of the scaffold. At the center of the colony, overlapping cells blurred the cell boundaries and projections of the peripheral cells were intertwined. The cells also created calcified nodules (Fig. 4C and D).

Histological analysis. A total of 2 weeks after transplantation into the rabbit ear, a large number of lymphocytes and erythrocytes could be seen near the border of the PLGA/BG scaffold, and the tissue arrangement in the scaffold was disordered (Fig. 5A and B). In PLGA/BG scaffolds 3 months post-transplantation, the numbers of lymphocytes and erythrocytes were markedly reduced than at 2 weeks and the composite scaffold was partly degraded. Histological analysis showed neovascularization in the transplanted area. The arrangement of fibers inside the infiltrating tissue began to align in parallel and the structure became denser. Additionally, the boundary between the graft material and the surrounding tissue became blurred (Fig. 5C and D). At 6 months post-transplantation, the PLGA/BG scaffold was fully degraded and had been replaced by newly grown fibers from the host, which were similar to the tissues surrounding the non-transplanted area (Fig. 5E and F).

Observation of Sirius red-stained paraffin sections under a polarized light microscope showed type I collagen fibers as red and yellow and type III collagen fibers as green. At 2 weeks post-transplantation, the area of green type III collagen fibers in the PLGA/BG scaffold was higher than the area of red type I collagen fibers (Fig. 6A). At 3 months post-transplantation, the area red type I collagen fibers in the transplanted area increased, and the area of the type III collagen fibers decreased in comparison with the result 2 weeks post-transplantation (Fig. 6B). The type I collagen fibers were the main collagen fibers in the graft areas. Type III collagen fibers were gradually replaced by red type I collagen fibers (Fig. 6C).

\section{Discussion}

The development of scaffolds with osteoinductivity, high porosity and stable degradation profiles is a key challenge in the field of tissue engineering. Although the relationship between pore size and bone formation is inconclusive, it is clear that porous structures provide more space and surface area for bone cell adhesion and bone growth (14). Simultaneously, the interconnection between pores provides a pathway for cell migration and vascularization in vivo (15-17). The scaffold used in the present study had a porous three-dimensional nanofibrous structure with 160-320 nm diameter fibers, a uniform pore distribution, and pore sizes of 1-7 $\mu \mathrm{m}$. In the in vitro experiment, the PLGA/BG nanofiber scaffold had a large surface area and provided a large number of cell contact points, which is beneficial to cell adhesion, invasion and proliferation (7). Studies have confirmed that nanofiber scaffolds are structurally closer to natural ECM, and are more conducive to cell adhesion and growth than traditional micron scaffolds $(18,19)$.

Expression of ALP is an early marker of a maturing extracellular matrix. It is an important marker for the in vitro 
A

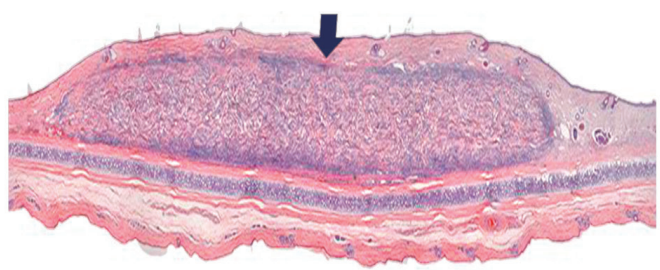

C

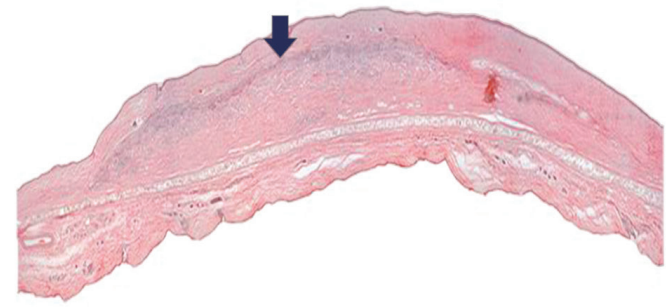

$\mathrm{E}$

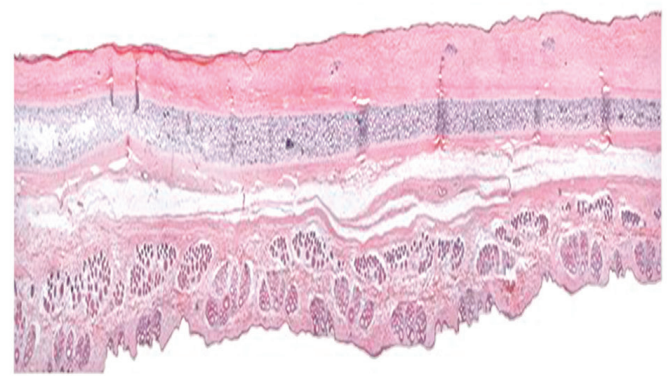

B

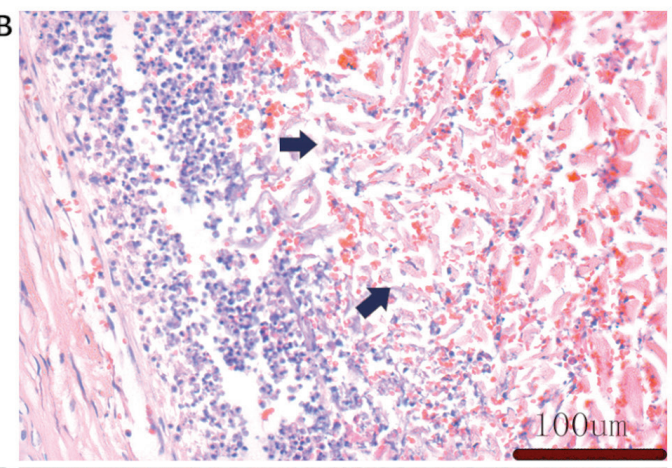

\section{D}
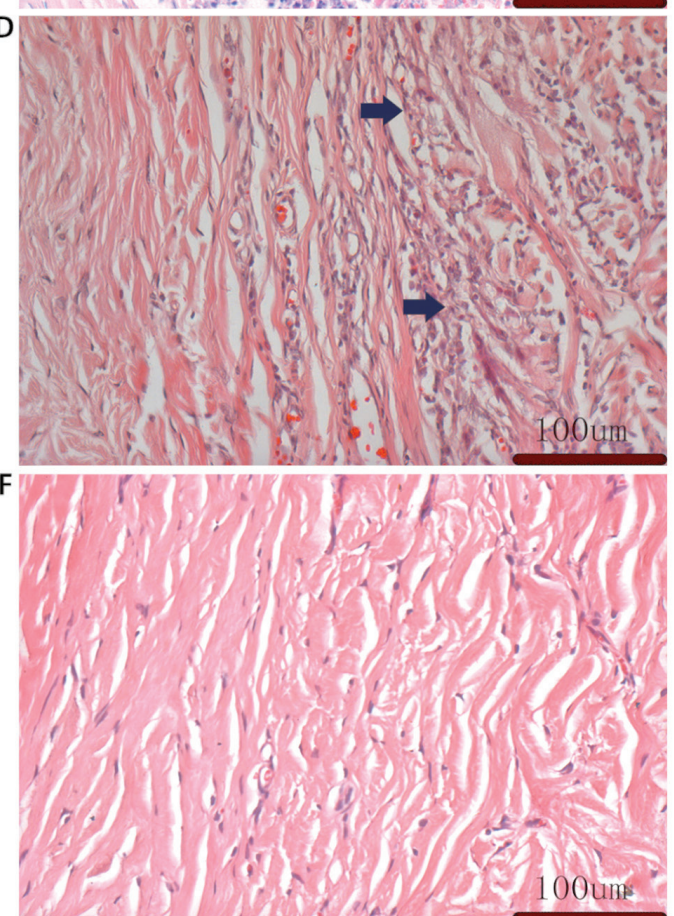

Figure 5. H\&E staining of the graft area. H\&E staining of the PLGA/BG scaffold graft area 2 weeks post-transplant at (A) $x 40$ and (B) $x 400$. H\&E staining of the PLGA/BG scaffold graft area at 3 months post-transplantation at (C) $x 40$ and (D) $x 400$. H\&E staining of the PLGA/BG scaffold graft area at 6 months post-transplantation, at (E) x40 and (F) x400. Arrows indicate the scaffold. BG, bioactive glass; PLGA, poly(lactic co-glycolic acid); H\&E, hematoxylin and eosin.
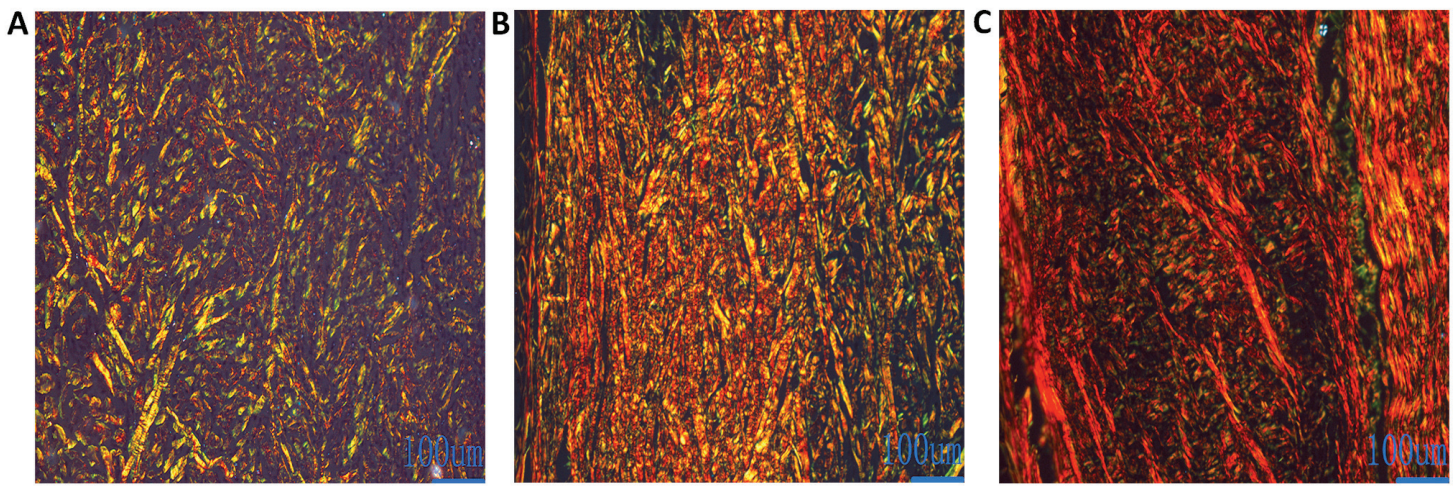

Figure 6. Sirius red staining of the PLGA/BG scaffold graft area. (A) Sirius red staining of the PLGA/BG scaffold graft 2 weeks post-transplant. (B) Sirius red staining of the PLGA/BG scaffold graft area at 3 months post-transplant. (C) Sirius red staining of the PLGA/BG scaffold graft area at 6 months post-transplant. PLGA, poly(lactic co-glycolic acid); BG, bioactive glass.

differentiation of osteoblasts into mature osteocytes that reach the matrix mineralization stage (9). In in vitro experiments, ALP is used as an early marker for osteoblast differentiation and maturation. The level of ALP secretion is positively correlated with the capacity for in vitro mineralization (20-22). It has previously been shown that bioactive glass that degrades in the early stage, releases calcium, phosphorus and silicon from the material into the plasma, and this stimulates the expression 
of ALP $(23,24)$. Studies such as that by Bellows et al $(25)$ have shown that each calcium nodule is formed by a nodular osteoblast that undergoes 2-3 weeks of proliferation and differentiation. The formation of calcified nodules is due to the multi-layer growth of osteoblasts, which must undergo three stages: Rapid proliferation, maturity of the extracellular matrix and mineralization of the matrix. Only after entering maturity, does mineralization occur. The results of SEM showed that, after 7 days of culture in the scaffold, cell proliferation and the number of calcified nodules increased gradually.

Stable degradation in vivo is a prerequisite for ideal bio-scaffolds. Premature degradation, or late or absent degradation, will affect bone remodeling, leading to the failure of bone defect repair. Novel scaffolds with good osteoconductivity and osteoinductivity have been prepared from PLGA and $\mathrm{BG}$, and their biocompatibility was previously reported in the literature (10-13). However, long-term studies on the stable degradation of PLGA/BG scaffolds in animals have, to the best of our knowledge, not yet been published.

In the animal experiments conducted in the present study, inflammatory reactions appeared in the PLGA/BG scaffold-transplanted area in the early post-operative period. A previous study has shown that the early inflammatory response is mediated by neutrophils releasing reactive oxygen species and inflammatory cytokines to clean the dead cells from the graft area (26). Additionally, neovascularization could be seen in the implanted areas in this present study. A previous study revealed that vascularization of the graft is not only key to graft survival, but that it is also important for osteogenesis. Mazio et al (27) aimed to obtain graft vascularization by promoting host vessel invasion of the scaffold, as well as by developing pre-vascularized constructs. An active blood vessel network helps grafts to survive and integrate with existing host tissue. Bone is a highly vascularized tissue that relies on tight connections between blood vessels and bone cells to maintain integrity (28). An inflammatory cell response could be observed in the transplant area and nascent immature type III collagen fibers were gradually replaced by type I collagen fibers, which were structurally and functionally more mature. Collagen is the most widespread fibrillar secretory protein in the body (29). It is a part of all tissues that require a framework or mechanical scaffold functionalized with growth factors, biological signals or specific structures allowing cells to adhere, proliferate and differentiate (29).

In the present study, the PLGA/BG scaffold had good biocompatibility and capacity for successful vascularization, and induced tissue maturation in the transplanted area. The scaffold could also be fully degraded after serving its purpose. The limitations of this study need to be further explored in future experiment. Results of the histological analysis are only qualitative in this study and detailed statistical analysis and comparison will be further studied. In future research, the physical and mechanical properties of the PLGA/BG scaffolds will also be further investigated as will cell adhesion based on primary human mesenchymal stem cells with the goal of examining the efficacy of the PLGA/BG scaffolds for the treatment of bone defects.

In summary, the PLGA/BG scaffold used in the current study had a three-dimensional network structure and a porosity of $93.048 \pm 0.121 \%$. The scaffold promoted cell adhesion, had good biocompatibility and provided a calcified matrix that promoted osteoblast formation. It may be a candidate bio-scaffold for the repair of bone defects.

\section{Acknowledgements}

Not applicable.

\section{Funding}

This work was supported by the Medical Science and Technology Research Fund Project of Guangdong Province (grant no. A2017547).

\section{Availability of data and materials}

The datasets used and/or analyzed during the present study are available from the corresponding author on reasonable request.

\section{Authors' contributions}

LY conceived and designed the study. SL performed the experiments and acquired the data. YC analyzed the data, prepared the figures and drafted the manuscript. JC and WF interpreted the results. WF edited and revised the manuscript. All authors have read and approved the final manuscript.

\section{Ethics approval and consent to participate}

All animal experiments in this study were approved by the Animal Ethical and Welfare Committee of Sun Yat-sen University.

\section{Patient consent for publication}

Not applicable.

\section{Competing interests}

The authors declare that they have no competing interests.

\section{References}

1. Furia JP, Rompe JD, Cacchio A and Maffulli N: Shock wave therapy as a treatment of nonunions, avascular necrosis, and delayed healing of stress fractures. Foot Ankle Clin 15: 651-662, 2010.

2. Lichte P, Pape HC, Pufe T, Kobbe P and Fischer H: Scaffolds for bone healing: Concepts, materials and evidence. Injury 42 : 569-573, 2011.

3. Palecek SP, Loftus JC, Ginsberg MH, Lauffenburger DA and Horwitz AF: Integrin-ligand binding properties govern cell migration speed through cell-substratum adhesiveness. Nature 385: 537-540, 1997.

4. Félix Lanao RP, Leeuwenburgh SC, Wolke JG and Jansen JA: Bone response to fast-degrading, injectable calcium phosphate cements containing PLGA microparticles. Biomaterials 32: 8839-8847, 2011.

5. Chereddy KK, Vandermeulen G and Préat V: PLGA based drug delivery systems: Promising carriers for wound healing activity. Wound Repair Regen 24: 223-236, 2016.

6. Gao S, Tang G, Hua D, Xiong R, Han J, Jiang S, Zhang Q and Huang C: Stimuli-responsive bio-based polymeric systems and their applications. J Mater Chem B Mater Biol Med 7: 709-729, 2019. 
7. Kido HW, Brassolatti P, Tim CR, Gabbai-Armelin PR, Magri AM, Fernandes KR, Bossini PS, Parizotto NA, Crovace MC, Malavazi I, et al: Porous poly (D,L-lactide-co-glycolide) acid/biosilicate ${ }^{\circledR}$ composite scaffolds for bone tissue engineering. J Biomed Mater Res B Appl Biomater 105: 63-71, 2017.

8. PeitlO,ZanottoED,SerbenaFCand Hench LL: Compositional and microstructural design of highly bioactive $\mathrm{P}_{2} \mathrm{O}_{5}-\mathrm{Na}_{2} \mathrm{O}-\mathrm{CaO}-\mathrm{SiO}_{2}$ glass-ceramics. Acta Biomater 8: 321-332, 2012.

9. Granito RN, Rennó AC, Ravagnani C, Bossini PS, Mochiuti D, Jorgetti V, Driusso P, Peitl O, Zanotto ED, Parizotto NA, et al: In vivo biological performance of a novel highly bioactive glass-ceramic (Biosilicate ${ }^{\circledR}$ ): A biomechanical and histomorphometric study in rat tibial defects. J Biomed Mater Res B Appl Biomater 97: 139-147, 2011

10. Habraken WJ, Wolke JG, Mikos AG and Jansen JA: PLGA microsphere/calcium phosphate cement composites for tissue engineering: In vitro release and degradation characteristics. J Biomater Sci Polym Ed 19: 1171-1188, 2008.

11. Ruhé PQ, Boerman OC, Russel FG, Mikos AG, Spauwen PH and Jansen JA: In vivo release of rhBMP-2 loaded porous calcium phosphate cement pretreated with albumin. J Mater Sci Mater Med 17: 919-927, 2006.

12. Ding J, Zhang J, Li J, Li D, Xiao C, Xiao H, Yang H, Zhuang X and Chen X: Electrospun polymer biomaterials. J.Prog Polym Sci 90: 1-34, 2019

13. Plachokova A, Link D, van den Dolder J, van den Beucken J and Jansen J: Bone regenerative properties of injectable PGLA-CaP composite with TGF-betal in a rat augmentation model. J Tissue Eng Regen Med 1: 457-464, 2007.

14. Gu BK, Choi DJ, Park SJ, Kim YJ and Kim CH: 3D bioprinting technologies for tissue engineering applications. Adv Exp Med Biol 1078: 15-28, 2018

15. Mastrogiacomo M, Scaglione S, Martinetti R, Dolcini L, Beltrame F, Cancedda R and Quarto R: Role of scaffold internal structure on in vivo bone formation in macroporous calcium phosphate bioceramics. Biomaterials 27: 3230-3237, 2006.

16. Deing A, Luthringer B, Laipple D, Ebel T and Willumeit R: A porous TiAl6V4 implant material for medical application. Int J Biomater 2014: 904230, 2014.

17. Chen G, Liu B, Liu H, Zhang H, Yang K, Wang Q, Ding J and Chang F: Calcium phosphate cement loaded with $10 \%$ vancomycin delivering high early and late local antibiotic concentration in vitro. Orthop Traumatol Surg Res 104: 1271-1275, 2018.

18. Jun HW, Paramonov SE, Dong H, Forraz N, McGuckin C and Hartgerink JD: Tuning the mechanical and bioresponsive properties of peptide-amphiphile nanofiber networks. J Biomater Sci Polym Ed 19: 665-676, 2008.

19. Murphy WL and Mooney DJ: Molecular-scale biomimicry. Nat Biotechnol 20: 30-31, 2002.
20. Proff P and Römer P: The molecular mechanism behind bone remodelling: A review. Clin Oral Investig 13: 355-362, 2009

21. Zhu B, Xu W, Liu J, Ding J and Chen X: Osteoinductive agents-incorporated three-dimensional biphasic polymer scaffold for synergistic bone pegeneration. J. ACS Biomater Sci Eng 5: 986-995, 2019.

22. Fávaro-Pípi E, Bossini P, de Oliveira P, Ribeiro JU, Tim C, Parizotto NA, Alves JM, Ribeiro DA, Selistre de Araújo HS and Renno AC: Low-intensity pulsed ultrasound produced an increase of osteogenic genes expression during the process of bone healing in rats. Ultrasound Med Biol 36: 2057-2064, 2010.

23. Renno AC, van de Watering FC, Nejadnik MR, Crovace MC, Zanotto ED, Wolke JG, Jansen JA and van den Beucken JJ: Incorporation of bioactive glass in calcium phosphate cement: An evaluation. Acta Biomater 9: 5728-5739, 2013.

24. Xynos ID, Edgar AJ, Buttery LD, Hench LL and Polak JM: Gene-expression profiling of human osteoblasts following treatment with the ionic products of Bioglass 45S5 dissolution. J Biomed Mater Res 55: 151-157, 2001.

25. Bellows CG, Aubin JE, Heersche JN and Antosz ME: Mineralized bone nodules formed in vitro from enzymatically released rat calvaria cell populations. Calcif Tissue Int 38: 143-154, 1986.

26. Liu K, Cai J, Li H, Feng J, Feng $\mathrm{C}$ and Lu F: The disturbed function of neutrophils at the early stage of fat grafting impairs long-term fat graft retention. Plast Reconstr Surg 142: 1229-1238, 2018.

27. Mazio C, Casale C, Imparato G, Urciuolo F, Attanasio C, De Gregorio M, Rescigno F and Netti PA: Pre-vascularized dermis model for fast and functional anastomosis with host vasculature. Biomaterials 192: 159-170, 2019.

28. Barabaschi GD, Manoharan V, Li Q and Bertassoni LE: Engineering pre-vascularized scaffolds for bone regeneration. Adv Exp Med Biol 881: 79-94, 2015.

29. Turkevych M, Turkevych A, Kadjaya A, Gold MH, Lotti T and Sulamanidze G: Pathomorphological criteria of use efficiency of resorbable and permanent implants in aesthetic medicine and cosmetic dermatology. J Cosmet Dermatol 17: 731-735, 2018.

This work is licensed under a Creative Commons Attribution-NonCommercial-NoDerivatives 4.0 International (CC BY-NC-ND 4.0) License. 